\title{
Simple Screened Hydrogen Model of Excitons in Two-Dimensional Materials
}

\author{
Olsen, Thomas; Latini, Simone; Rasmussen, Filip Anselm; Thygesen, Kristian Sommer
}

Published in:

Physical Review Letters

Link to article, DOI:

10.1103/PhysRevLett.116.056401

Publication date:

2016

Document Version

Publisher's PDF, also known as Version of record

Link back to DTU Orbit

Citation (APA):

Olsen, T., Latini, S., Rasmussen, F. A., \& Thygesen, K. S. (2016). Simple Screened Hydrogen Model of Excitons in Two-Dimensional Materials. Physical Review Letters, 116(5), [056401].

https://doi.org/10.1103/PhysRevLett.116.056401

\section{General rights}

Copyright and moral rights for the publications made accessible in the public portal are retained by the authors and/or other copyright owners and it is a condition of accessing publications that users recognise and abide by the legal requirements associated with these rights.

- Users may download and print one copy of any publication from the public portal for the purpose of private study or research.

- You may not further distribute the material or use it for any profit-making activity or commercial gain

- You may freely distribute the URL identifying the publication in the public portal

If you believe that this document breaches copyright please contact us providing details, and we will remove access to the work immediately and investigate your claim 


\title{
Simple Screened Hydrogen Model of Excitons in Two-Dimensional Materials
}

\author{
Thomas Olsen, ${ }^{*}$ Simone Latini, Filip Rasmussen, and Kristian S. Thygesen \\ Center for Atomic-Scale Materials Design and Center for Nanostructured Graphene (CNG), \\ Department of Physics, Technical University of Denmark, 2800 Kgs. Lyngby, Denmark
}

(Received 22 October 2015; published 2 February 2016)

\begin{abstract}
We present a generalized hydrogen model for the binding energies $\left(E_{B}\right)$ and radii of excitons in twodimensional (2D) materials that sheds light on the fundamental differences between excitons in two and three dimensions. In contrast to the well-known hydrogen model of three-dimensional (3D) excitons, the description of $2 \mathrm{D}$ excitons is complicated by the fact that the screening cannot be assumed to be local. We show that one can consistently define an effective $2 \mathrm{D}$ dielectric constant by averaging the screening over the extend of the exciton. For an ideal 2D semiconductor this leads to a simple expression for $E_{B}$ that only depends on the excitonic mass and the 2D polarizability $\alpha$. The model is shown to produce accurate results for 51 transition metal dichalcogenides. Remarkably, over a wide range of polarizabilities the binding energy becomes independent of the mass and we obtain $E_{B}^{2 D} \approx 3 /(4 \pi \alpha)$, which explains the recently observed linear scaling of exciton binding energies with band gap. It is also shown that the model accurately reproduces the nonhydrogenic Rydberg series in $\mathrm{WS}_{2}$ and can account for screening from the environment.
\end{abstract}

DOI: 10.1103/PhysRevLett.116.056401

A striking property of two-dimensional semiconductors is the ability to host strongly bound excitons. This was initially predicted theoretically for hexagonal boron nitride $(\mathrm{hBN})$ [1], graphane [2], and various transition metal dichalcogenides [3-5], and has subsequently been confirmed experimentally [6-8]. The quantum confinement of excitons in two dimensions comprises a tempting and intuitively appealing explanation for the large binding energies in these materials [9]. However, it has become clear that it is the reduced dielectric screening in two dimensions that is the main origin of the large binding energy $[3,10]$. The $2 \mathrm{D}$ electronic system is rather poor at screening interactions and the effective Coulomb interaction between an electron and a hole is simply much stronger in two dimensions than in three dimensions.

A rigorous treatment of excitons requires advanced computational methodology such as the Bethe-Salpeter equation $[11,12]$. This approach has been applied to obtain absorption spectra for numerous insulators and usually yields very good agreement with experiments [13]. However, only systems of modest size can be treated by such methods and simplified models of excitons will be an inevitable ingredient in calculations of realistic systems. For example, if the effect of substrates or the dielectric environment is to be included in the calculation of excitons in 2D systems [14], the computations become intractable with a standard Bethe-Salpeter approach. For 3D materials the Mott-Wannier model comprises a strong conceptual and intuitive picture that provides a simple framework for calculating exciton binding energies [15]. In the centerof-mass frame, an excited electron-hole pair can be shown to satisfy a hydrogenic Schrödinger equation, where band structure effects are included through an excitonic effective mass $\mu$ and the dielectric screening from the environment is included through the static dielectric constant $\epsilon_{0}$. The exciton binding energy in atomic units is then written as

$$
E_{B}^{3 \mathrm{D}}=\frac{\mu}{2 \epsilon_{0}^{2}} .
$$

Thus, the daunting task of solving the Bethe-Salpeter equation, has been reduced to the calculation of just two parameters: the effective mass and the static dielectric constant, both of which are easily obtained with any standard electronic structure software package. This approximation is well justified whenever the screening is local, such that its Fourier transform can be approximated by a constant in the vicinity of the origin. However, in highly anisotropic structures such as layered materials this assumption is expected to break down.

In $2 \mathrm{D}$ dielectrics, it is well known that the screening takes the form $\epsilon(\mathbf{q})=1+2 \pi \alpha q$ [2], where $\alpha$ is the 2D polarizability. The screening is thus inherently nonlocal in real space, and it is not obvious if it is possible to arrive at a hydrogenic model like Eq. (1). Instead, one can calculate the 2D screened potential and solve the Schrödinger equation for the electron-hole wave function

$$
\left[-\frac{\nabla^{2}}{2 \mu}+W(\mathbf{r})\right] \psi(\mathbf{r})=E_{n} \psi(\mathbf{r})
$$

where $W(\mathbf{r})$ is the $2 \mathrm{D}$ convolution of the Coulomb interaction and $\epsilon^{-1}\left(\mathbf{r}-\mathbf{r}^{\prime}\right)$. This approach has previously been shown to provide good agreement with the BetheSalpeter equation [14,16]. In the Supplemental Material 
[17] we assess that the binding energies of 7 transition metal dichalcogenides obtained with the Bethe-Salpeter equation agree well with results obtained from Eq. (2). However, in general the solution of Eq. (2) is a tedious task and it would be highly desirable to have an expression like Eq. (1) from which the exciton binding energy in a given material can be easily estimated and understood. To accomplish this, we calculate the average screening felt by the exciton. To this end, we consider the expression

$$
\epsilon_{\mathrm{eff}}=\frac{a_{\mathrm{eff}}^{2}}{\pi} \int_{0}^{2 \pi} d \theta \int_{0}^{1 / a_{\mathrm{eff}}} d q q \epsilon(\mathbf{q})
$$

where $a_{\mathrm{eff}}$ is the effective Bohr radius. For the 2D hydrogen atom the Bohr radius is given by $a=\epsilon /(2 \mu)$ and Eq. (4) has to be solved self-consistently for $\epsilon_{\text {eff }}$ given an expression for $\epsilon(\mathbf{q})$. In a strictly 2D system, the screening is linear in $q$ and Eq. (3) can be solved to yield

$$
\epsilon_{\mathrm{eff}}=\frac{1}{2}(1+\sqrt{1+32 \pi \alpha \mu / 3}) .
$$

Using that the hydrogenic binding energy in two dimensions is a factor of four larger than in three dimensions [9], we obtain

$$
E_{B}^{2 \mathrm{D}}=\frac{8 \mu}{(1+\sqrt{1+32 \pi \alpha \mu / 3})^{2}} .
$$

This is the main result of the present Letter and comprises a long-sought-for 2D analog of Eq. (1).

A remarkable property of the expression (5) is the fact that it becomes independent of the effective mass if the polarizability is large. More precisely,

$$
E_{B}^{2 \mathrm{D}} \approx \frac{3}{4 \pi \alpha}, \quad 32 \pi \alpha \mu / 3 \gg 1 .
$$

It may come as a surprise that the binding energy becomes independent of mass, since a large mass gives rise to a localized exciton and the binding energy typically increases with localization. This is reflected in Eq. (1), where the binding energy is seen to be proportional to the mass. However, in two dimensions, short range interactions are screened more effectively than long range interactions. Thus, there are two opposing effects of the exciton mass and for large polarizabilities the binding energy becomes independent of mass. In order to assert the applicability of the expressions (5)-(6), we have calculated the effective masses and static polarizabilities (in the random phase approximation) of 51 semiconducting monolayers of transition metal dichalcogenides. For indirect band gap materials we use the effective mass at the indirect gap. The calculations were performed with the electronic structure code GPAW [18,19], and we refer to the Supplemental Material [17] and Ref. [20] for details on the calculations.

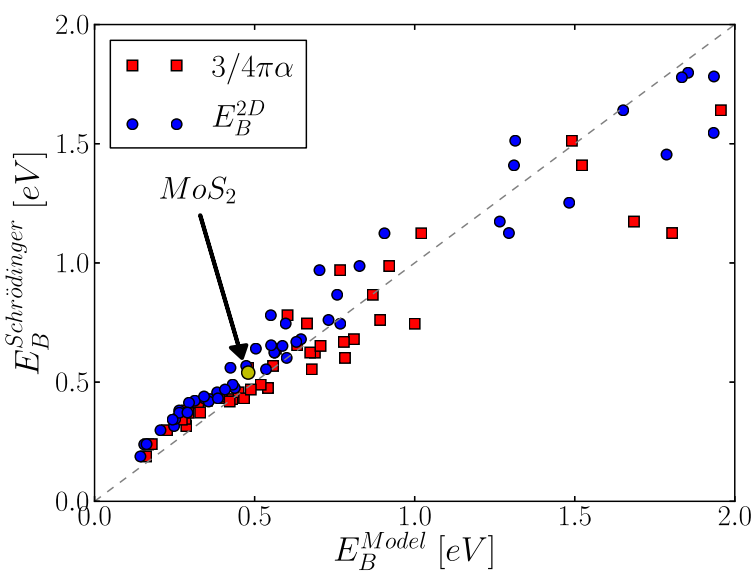

FIG. 1. Exciton binding energies of 51 transition metal dichalcogenides calculated as the lowest eigenvalue of Eq. (2) (vertical axis) and the model result Eq. (5) (horizontal axis). We have indicated the well-known example of $\mathrm{MoS}_{2}$.

In Fig. 1 we compare the model binding energies with the full solution of Eq. (2). Using the expression (5), the agreement is seen to be on the order of $10 \%$. With the approximated expression (6), we obtain excellent agreement for binding energies up to $\sim 0.5 \mathrm{eV}$, whereas the binding energies are underestimated for strongly bound excitons.

Recently, first-principles calculations have indicated that exciton binding energies in different 2D materials scale linearly with the band gaps [21]. In the present model, this behavior comes out naturally since (without local field effects) the in-plane components of the polarizability in the random phase approximation are given by

$$
\alpha=\sum_{m, n} \int_{B Z} \frac{d \mathbf{k}}{(2 \pi)^{2}}\left(f_{n \mathbf{k}}-f_{m \mathbf{k}}\right) \frac{\left|\left\langle u_{m \mathbf{k}}\left|\hat{r}_{\|}\right| u_{n \mathbf{k}}\right\rangle\right|^{2}}{\varepsilon_{n \mathbf{k}}-\varepsilon_{m \mathbf{k}}},
$$

and we expect that $\alpha$ will be roughly inversely proportional to the band gap. This is illustrated in Fig. 2 for the 51

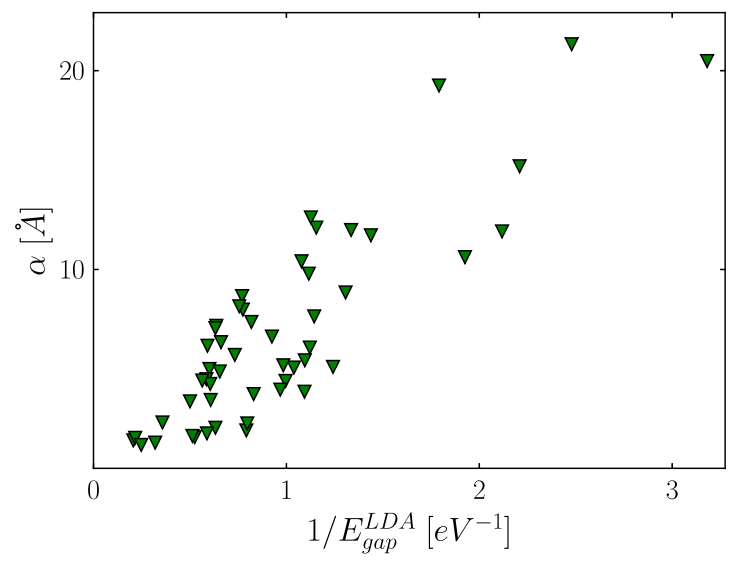

FIG. 2. The 2D polarizability of 51 transition metal dichalcogenides shown as a function of LDA band gaps. 
transition metal dichalcogenides. Combining this with Eq. (6) thus gives $E_{B}^{2 \mathrm{D}} \propto E_{\text {gap }}$. However, in the present model the scaling originates solely from the screening and not the effective mass as previously proposed [21]. For the present set of materials, we do not observe any correlation between binding energies and effective mass. We use the LDA band gaps and not the quasiparticle gaps, which could be obtained from, for example, $G W$ calculations [20], since LDA typically gives a better estimate of the two-particle excitation energies that enters the expression for $\alpha$. In contrast, the use of $G W$ gaps would underestimate the screening due to the lack of electron-hole interactions.

To validate the general applicability of the effective screening model, we now show that it can also be used to account for the entire exciton spectrum in 2D materials. In Ref. [22], the exciton spectra of graphene derivatives was predicted to deviate from the 2D Rydberg series and in Ref. [23], the exciton spectrum of $\mathrm{WS}_{2}$ was measured and shown to deviate significantly from the Rydberg series of a 2D hydrogen model scaled by an overall screening factor. The reason is simply that the effective screening depends on the $n$ quantum number due to the increasing spatial extent of higher lying Rydberg states. The authors used the results to define $n$-dependent effective screenings $\epsilon_{n}$, which were then determined by fitting each term in the Rydberg series to a 2D hydrogen model. The Rydberg series is thus written as

$$
E_{n}^{2 \mathrm{D}}=-\frac{\mu}{2\left(n-\frac{1}{2}\right)^{2} \epsilon_{n}^{2}}
$$

Two of the present authors have recently shown that the Rydberg series can accurately be reproduced by solving Eq. (2) with a screened 2D potential calculated from first principles [14], and we will assume that approach to be an accurate reference. Here we calculate the $n$-dependent effective screening from first principles by replacing $a_{\text {eff }}$ in Eq. (3) by an $n$-dependent characteristic extension of the state. To this end, we note that for $l=0$, the first moment of a state with principal quantum number $n$ in a 2D hydrogen atom with Coulomb interaction scaled by $1 / \epsilon$ is [9]

$$
a_{n} \equiv\langle n|\hat{r}| n\rangle=\epsilon[3 n(n-1)+1] /(2 \mu),
$$

where $\hat{r}=\sqrt{\hat{x}^{2}+\hat{y}^{2}}$. In terms of this, the $a_{\text {eff }}$ defined previously is given by $a_{1}$ and $E_{B}^{2 \mathrm{D}}$ is $-E_{1}^{2 \mathrm{D}}$. Within the linear model the effective screening for state $n$ then becomes

$$
\epsilon_{n}=\frac{1}{2}\left(1+\sqrt{1+\frac{32 \pi \alpha \mu}{9 n(n-1)+3}}\right) .
$$

It is straightforward to generalize these expressions to $l \neq 0$ [9], which results in a larger value of the effective radius $a_{n l}$ and thus $\epsilon_{n, l>0}<\epsilon_{n, l=0}$. The energy is still given by Eq. (8) and at a given $n$, the higher angular momentum excitons will therefore have a larger binding energy, which has been observed in the case of $2 \mathrm{H}-\mathrm{WS}_{2}$ monolayers [6]. As a case study we consider this material and apply the linear screening model. We obtain a first-principles 2D polarizability of $\alpha=5.25 \AA$ and $\mu=0.19$. In Fig. 3 we show the Rydberg series calculated with the generalized hydrogen model, which agrees very well with a full solution of Eq. (2). In contrast, the pure 2D hydrogen model with an overall effective screening is seen to significantly underestimate the binding energies at higher lying states, since the decreased screening of extended states is not taken into account. We also note that the model binding energies of the $n=1$ state agree very well with a full solution of the Bethe-Salpeter equation, which yields an exciton binding energy of $0.54 \mathrm{eV}$ [24].

We now proceed to show how the effect of screening by the environment can naturally be taken into account in the present framework. It should be noted, however, that the linear model for the screening is expected to break down for systems where the vertical extent of a substrate becomes comparable to the Bohr radius of the exciton. For example, if we consider a stack of $N$ monolayers, $\alpha$ will diverge in the limit of large $N$, since the bulk system will have $\epsilon(\mathbf{q}=\mathbf{0}) \neq 1[14,25]$. The linear regime will therefore only be valid for $q \ll 1 / N d$, where $d$ is the interlayer distance. As an example where we expect the linear model to be applicable, we consider a monolayer $2 \mathrm{H}-\mathrm{MoS}_{2}$ and compare the isolated layer with the two cases where it is in the vicinity of another layer of $2 \mathrm{H}-\mathrm{MoS}_{2}$ and in the vicinity of a metallic layer of $1 T-\mathrm{MoS}_{2}$. In Fig. 4, we show the absorption spectrum calculated from the Bethe-Salpeter equation based on Kohn-Sham eigenvalues. The BSE calculations were performed in a plane wave basis with a 2D Coulomb truncation scheme [26,27] using a $60 \times 60$ $k$-point mesh. It is well known that the low energy absorption spectrum of this system exhibits a double

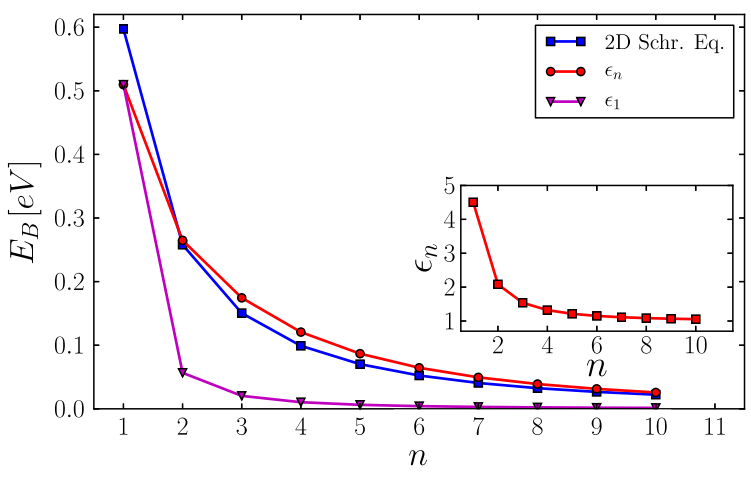

FIG. 3. Rydberg series of a monolayer of $2 \mathrm{H}-\mathrm{WS}_{2}$ calculated with the generalized hydrogen model with linear screening [Eqs. (8) and (10)] and from the solution of the 2D screened Schrödinger equation (2). The results are compared with the bare hydrogen model where the effective screening obtained from the ground states is used for all states. 


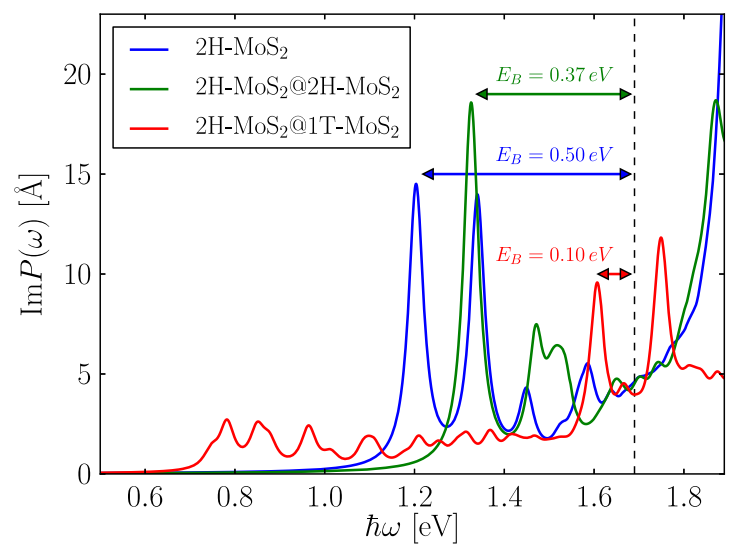

FIG. 4. Dynamic 2D polarizability of $2 \mathrm{H}-\mathrm{MoS}_{2}$ in different environments calculated from the Bethe-Salpeter equation based on Kohn-Sham eigenvalues. The vertical lines at $1.7 \mathrm{eV}$ marks the Kohn-Sham band gaps, which are nearly identical in the three cases.

excitonic peak due a spin-orbit split valence band [28,29]. This facilitates the identification of the excitons in the $2 \mathrm{H}-\mathrm{MoS}_{2}$ layer in the vicinity of a metallic substrate with low lying excitations. We have not performed the full spinorial BSE calculations, but simply included spin-orbit effects in the band structure in order to identify the excitons. In the following we consider the binding energies of the lowest exciton. The isolated layer exhibits an exciton bound by $0.50 \mathrm{eV}$. In the vicinity of another $2 \mathrm{H}-\mathrm{MoS}_{2}$ layer, the binding energy is decreased to $0.37 \mathrm{eV}$ and the metallic $1 T-\mathrm{MoS}_{2}$ decreases the binding energy to $0.10 \mathrm{eV}$. We note that the quasiparticle band structure corrections are expected to be much smaller for the case of $2 \mathrm{H}-\mathrm{MoS}_{2} @ 1 T-\mathrm{MoS}_{2}$ such that the actual positions of the excitons would be similar for the three cases in an optical absorption experiment. However, we have chosen to leave out the quasiparticle corrections in order to illustrate the difference in binding energies more clearly.

To apply the model we wish to calculate $\epsilon(\mathbf{q})$ for the $2 \mathrm{H}-\mathrm{MoS}_{2}$ layer when it is in the vicinity of a screening environment. For small $q$, we may still write it as $\epsilon(\mathbf{q})=$ $1+2 \pi \tilde{\alpha} q$ and we would like to extract $\tilde{\alpha}$, which is the relevant quantity for the screened hydrogen model. We calculate it by the finite difference

$$
2 \pi \tilde{\alpha}=\epsilon\left(\mathbf{q}_{1}\right)-1,
$$

where $\mathbf{q}_{1}$ is a small finite value of $q$. In the present case we take $\mathbf{q}_{1}$ as the smallest $q$ vector in the direction of $K$ obtained from a $60 \times 60 k$-point grid. The $2 \mathrm{D}$ dielectric function is obtained from

$$
\frac{1}{\epsilon(\mathbf{q})}=\frac{\left\langle V_{\mathrm{tot}}\left(r_{\|}, z_{0}\right) e^{-i \mathbf{q} \cdot \mathbf{r}}\right\rangle_{A}}{V_{\mathbf{q}}}
$$

where $V_{\text {tot }}(\mathbf{r})$ is the total potential resulting from an external perturbation $V_{\text {ext }}(\mathbf{r})=V_{\mathbf{q}} e^{i \mathbf{q} \cdot \mathbf{r}}$ and $\langle\ldots\rangle_{A}$ denotes the average over the $2 \mathrm{D}$ unit cell of area $A$. It is straightforward to relate this expression to an average over the microscopic dielectric function $\epsilon^{-1}\left(\mathbf{r}, \mathbf{r}^{\prime}\right)$, which can be calculated in the random phase approximation by most electronic structure codes. We take $z_{0}$ to be at the center of the $2 \mathrm{H}-\mathrm{MoS}_{2}$ layer, but we note that $\tilde{\alpha}$ is approximately independent of the value of $z_{0}$ when $z_{0}$ is chosen in any part of the central $3.0 \AA$ of the layer. In Table I, we display the calculated values of $\tilde{\alpha}$ along with the exciton binding energies obtained from the model (5), the 2D Schrödinger equation (2), and the BSE calculations. As expected, the environment strongly affects the value of $\tilde{\alpha}$. In particular, the metallic $1 T-\mathrm{MoS}_{2}$ layer significantly increases the screening, whereas the presence of another $2 \mathrm{H}-\mathrm{MoS}_{2}$ layer results in a less pronounced effect. We find good agreement between the simple model, the 2D Schrödinger equation, and the BSE calculations. We should note that the convergence of the exciton binding energies in the presence of the metallic $1 T-\mathrm{MoS}_{2}$ layer is very slow with respect to $k$-point sampling and the converged result is expected to exhibit a lower binding energy than the one obtained here. Furthermore, we have not included the intraband contribution (Drude response) to the static screening, which is expected to scale as $\sim 1 / q$ in $2 \mathrm{D}$ metals. On the other hand, the $1 T$ structure is known to distort into the so-called $1 T^{\prime}$ structure, which is a topological insulator with a gap on the order $50 \mathrm{meV}$ [30], and in that case the Drude response will not be present. In any case, the screening is treated at the same footing in the BSE and the model calculations since the values of $\tilde{\alpha}$ were obtained by a finite difference calculation on the same $k$-point grid that was used in the

TABLE I. Exciton binding energies for $2 \mathrm{H}-\mathrm{MoS}_{2}$ in different environments calculated from the Bethe-Salpeter equation (BSE), the 2D Schrödinger equation, and the generalized screened hydrogen model. We also display the values of $\tilde{\alpha}$, which is the polarizability of the single $2 \mathrm{H}-\mathrm{MoS}_{2}$ layer used in the calculations. For all calculations we used an effective exciton mass of 0.276 , which was obtained from the $a b$ initio band structure.

\begin{tabular}{lccc}
\hline \hline & $2 H-\mathrm{MoS}_{2}$ & $2 H-\mathrm{MoS}_{2} @ 2 H-\mathrm{MoS}_{2}$ & $2 H-\mathrm{MoS}_{2} @ 1 T-\mathrm{MoS}_{2}$ \\
\hline$E_{B}^{\text {BSE }}[\mathrm{eV}]$ & 0.50 & 0.37 & 0.10 \\
$E_{B}^{\text {Schr }} \cdot[\mathrm{eV}]$ & 0.54 & 0.40 & 0.17 \\
$E_{B}^{\mathrm{Model}}[\mathrm{eV}]$ & 0.48 & 0.30 & 0.10 \\
$\tilde{\alpha}[\AA]$ & 5.83 & 10.0 & 30.1 \\
\hline \hline
\end{tabular}


BSE calculations. The exact conditions under which the linear model is applicable will depend on the thickness of the substrate as well as the screening properties of the substrate. For extended substrates, the present approach may be generalized by calculating the full $\epsilon(\mathbf{q})$ and solving Eq. (3) numerically, but it is not clear that the analytical results derived from the 2D hydrogen model (8) are able to produce reliable results in this case. Alternatively, one may solve a quasi-2D Schrödinger equation that incorporates the finite extent of the slab [25]. We note that the present method can be viewed as a generalized hydrogen model analogue of the approach taken by Ugeda et al. [7], where the full substrate screening was taken into account when solving the Bethe-Salpeter equation for the layer.

To conclude, we have presented an analytical expression for the exciton binding energies in 2D semiconductors that only depends on the static 2D polarizability and the effective mass, and produces good agreement with the solution of the full screened 2D Schrödinger equation. It has also been shown that for large polarizabilities, the result becomes independent of mass and yields a linear relation between exciton binding energies and band gaps. It has previously been anticipated that the nonhydrogenic Rydberg series could be attributed to an $n$-dependent value of the effective screening [23]. Here we have obtained an explicit expression for $\epsilon_{n}$ that provides an accurate account of the full exciton spectrum. It has also been shown that the model can be generalized to incorporate the effect of a simple screening environment. We do not claim that the presented expression for the effective screening (3) in the linear model is unique. In fact, it is based on an unweighted average of a linear model for the nonlocal 2D screening over the extent of the exciton and it is easy to imagine more elaborate averaging schemes. However, we believe that the simplicity is the main merit of this procedure and the resulting analytical expressions are very easy to apply to a given 2D material. In particular, for complicated structures it may not be possible to treat the electron-hole interaction by a first-principles approach and our model results could be a crucial ingredient in understanding the excitonic structure in such materials.

The Center for Nanostructured Graphene (CNG) is sponsored by the Danish National Research Foundation, Project No. DNRF58.

*tolsen@fysik.dtu.dk

[1] L. Wirtz, A. Marini, and A. Rubio, Phys. Rev. Lett. 96, 126104 (2006).

[2] P. Cudazzo, C. Attaccalite, I. V. Tokatly, and A. Rubio, Phys. Rev. Lett. 104, 226804 (2010).

[3] F. Hüser, T. Olsen, and K. S. Thygesen, Phys. Rev. B 88, 245309 (2013).
[4] H. P. Komsa and A. V. Krasheninnikov, Phys. Rev. B 86, 241201 (2012).

[5] D. Y. Qiu, F. H. da Jornada, and S. G. Louie, Phys. Rev. Lett. 111, 216805 (2013).

[6] Z. Ye, T. Cao, K. OBrien, H. Zhu, X. Yin, Y. Wang, S. G. Louie, and X. Zhang, Nature (London) 513, 214 (2014).

[7] M. M. Ugeda, A. J. Bradley, S.-F. Shi, F. H. da Jornada, Y. Zhang, D. Y. Qiu, W. Ruan, S.-K. Mo, Z. Hussain, Z.-X. Shen, F. Wang, S. G. Louie, and M. F. Crommie, Nat. Mater. 13, 1091 (2014).

[8] A. Hanbicki, M. Currie, G. Kioseoglou, A. Friedman, and B. Jonker, Solid State Commun. 203, 16 (2015).

[9] X. L. Yang, S. H. Guo, F. T. Chan, K. W. Wong, and W. Y. Ching, Phys. Rev. A 43, 1186 (1991).

[10] P. Cudazzo, I. V. Tokatly, and A. Rubio, Phys. Rev. B 84, 085406 (2011).

[11] S. Albrecht, L. Reining, R. Del Sole, and G. Onida, Phys. Rev. Lett. 80, 4510 (1998).

[12] M. Rohlfing and S. G. Louie, Phys. Rev. Lett. 81, 2312 (1998).

[13] G. Onida, L. Reining, and A. Rubio, Rev. Mod. Phys. 74, 601 (2002).

[14] K. Andersen, S. Latini, and K. S. Thygesen, Nano Lett. 15 4616 (2015).

[15] G. H. Wannier, Phys. Rev. 52, 191 (1937).

[16] T. C. Berkelbach, M.S. Hybertsen, and D. R. Reichman, Phys. Rev. B 88, 045318 (2013).

[17] See Supplemental Material at http://link.aps.org/ supplemental/10.1103/PhysRevLett.116.056401 for computational details and a comparison of the 2D Scrödinger equation with BSE calculations for 7 TMDs.

[18] J. Enkovaara et al., J. Phys. Condens. Matter 22, 253202 (2010).

[19] J. Yan, J. J. Mortensen, K. W. Jacobsen, and K. S. Thygesen, Phys. Rev. B 83, 245122 (2011).

[20] F. A. Rasmussen and K. S. Thygesen, J. Phys. Chem. C 119, 13169 (2015).

[21] J.-H. Choi, P. Cui, H. Lan, and Z. Zhang, Phys. Rev. Lett. 115, 066403 (2015)

[22] S. Huang, Y. Liang, and L. Yang, Phys. Rev. B 88, 075441 (2013).

[23] A. Chernikov, T. C. Berkelbach, H. M. Hill, A. Rigosi, Y. Li, O. B. Aslan, D. R. Reichman, M. S. Hybertsen, and T. F. Heinz, Phys. Rev. Lett. 113, 076802 (2014).

[24] H. Shi, H. Pan, Y.-W. Zhang, and B. I. Yakobson, Phys. Rev. B 87, 155304 (2013).

[25] S. Latini, T. Olsen, and K. S. Thygesen, Phys. Rev. B 92, 245123 (2015).

[26] C. A. Rozzi, D. Varsano, A. Marini, E. K. U. Gross, and A. Rubio, Phys. Rev. B 73, 205119 (2006).

[27] F. Hüser, T. Olsen, and K. S. Thygesen, Phys. Rev. B 87, 235132 (2013).

[28] K. F. Mak, C. Lee, J. Hone, J. Shan, and T. F. Heinz, Phys. Rev. Lett. 105, 136805 (2010).

[29] A. Molina-Sánchez, D. Sangalli, K. Hummer, A. Marini, and L. Wirtz, Phys. Rev. B 88, 045412 (2013).

[30] X. Qian, J. Liu, L. Fu, and J. Li, Science 346, 1344 (2014). 